EPJ Web of Conferences 64, 02007 (2014)

DOI: $10.1051 /$ epjconf/ 20146402007

(C) Owned by the authors, published by EDP Sciences, 2014

\title{
Simulation of cyclotron resonant scattering features
}

\section{The effect of bulk velocity}

\author{
Fritz-Walter Schwarm ${ }^{1,2, a}$, G. Schönherr ${ }^{3}$, M. Kühnel ${ }^{1,2}$, and J. Wilms ${ }^{1,2}$ \\ ${ }^{1}$ Dr. Karl Remeis-Sternwarte Bamberg, Sternwartstraße 7, 96049 Bamberg, Germany \\ ${ }^{2}$ Erlangen Centre for Astroparticle Physics (ECAP), Erwin-Rommel-Str. 1, 91058 Erlangen, Germany \\ ${ }^{3}$ Leibniz-Institut für Astrophysik Potsdam, An der Sternwarte 16, 14482 Potsdam, Germany
}

\begin{abstract}
X-ray binary systems consisting of a mass donating optical star and a highly magnetized neutron star, under the right circumstances, show quantum mechanical absorption features in the observed spectra called cyclotron resonant scattering features (CRSFs). We have developed a simulation to model CRSFs using Monte Carlo methods. We calculate Green's tables which can be used to imprint CRSFs to arbitrary X-ray continua. Our simulation keeps track of scattering parameters of individual photons, extends the number of variable parameters of previous works, and allows for more flexible geometries. Here we focus on the influence of bulk velocity of the accreted matter on the CRSF line shapes and positions.
\end{abstract}

\section{Introduction}

Electrons in the vicinity of strong magnetic fields on the order of some $10^{12} \mathrm{G}$, such as the ones of some neutron star binary systems, couple to the magnetic field lines and therefore funnel down the magnetic poles assuming a dipolar field on the corresponding length scales. When the magnetic field strength becomes comparable to a few percent of the critical magnetic field, $B_{\text {crit }}=44.14 \cdot 10^{12} \mathrm{G}$, the electrons populate discrete energy states perpendicular to the magnetic field, the Landau levels. The processes responsible for the formation of cyclotron lines as observed in some of these systems are the excitation of electrons from one level to another one and resonant scattering without level transitions. These are described by complex relativistic scattering cross sections which can be calculated analytically, although with some computational effort. The situation becomes more complicated if one considers the continuous relativistic momentum distribution of the electrons parallel to the magnetic field characterized by the plasma temperature. Since the parallel movement of the electrons alters their angle and energy in the electron rest frame the resonances are effectively broadened. Additionally there is another velocity component playing a non-negligible role here which is the bulk velocity of the electrons at the height of the line forming region. This velocity depends on the free fall velocity and the radiative pressure at the altitude of interest (see [1]) and leads to another boosting factor for the incoming photon. Since the mean free path of a photon depends on the scattering electron's momentum parallel to the magnetic field, an integration over a suitable momentum distribution is needed for the sampling of scattering parameters in Monte Carlo simulations. This integration can

a e-mail: Fritz.Schwarm@sternwarte.uni-erlangen.de 
not be performed analytically therefore we use a numerical adaptive Simpson integration method to calculate the corresponding partial integrals in conjunction with a table interpolation scheme.

\section{Simulation}

Photons generated by various continuum processes such as cyclotron and blackbody radiation interact with infalling electrons moving with relativistic velocities towards the hot spot on the surface of the neutron star. Photons interacting with an electron may excite it to a higher Landau level thereby being absorbed or scattered into another spectral regime. This way cyclotron resonant absorption features are formed. Their shape is further altered by secondary photons spawned by electron deexcitations which happen to consist of mainly single level transitions from Landau level $n$ to $n^{\prime}=n-1$ [2]. The energy difference between these Landau levels depends on the scattering angle and the electron's parallel momentum but roughly agrees with the first resonance. Therefore spawned photons tend to fill up the fundamental cyclotron line making it a complex shaped feature possibly even with line wings seen in emission. Monte Carlo simulations of CRSFs provide a straight forward way to obtain the exact line shape of this complex fundamental cyclotron line and a reasonable amount of harmonics with computationally handable effort. Such Monte Carlo simulations have been performed by a variety of authors ([3],[4],[5]). Here we discuss the influence of the electrons' bulk velocity on the line shape. The simulation we are using is built on similar methods and follows the same physical formulation as utilized and described by [4] while the usage of the Green's functions approach as it was introduced by [5] allows for the input of arbitrary continua. This new code has technically been set up completely from scratch in order to yield the desired flexibility in geometry and physical setting. In particular any distribution of the magnetic field strength, density, temperature, and bulk velocity can be assigned to the simulated line forming region. Here we discuss the effect of the motion of the hot infalling plasma on cyclotron resonant scattering processes of X-ray photons and plasma electrons. We simulate CRSFs in X-ray spectra by generating photons according to a certain seed photon continuum within the column and propagating them through a CRSF medium with plasma temperature $T$ parallel to the magnetic field and bulk velocity $v_{\text {bulk. }}$. The neutron star reference frame in which the surface is at rest will be referred to as neutron star or lab frame. All figures are shown in this frame as it would correspond to the observer frame for a theoretical non spinning non orbiting neutron star. The rest or plasma frame has a relative velocity of $v_{\text {bulk }}$ with respect to the neutron star frame.

\section{Bulk velocity and its influence on CRSFs}

All calculations are done in the electrons' rest frame. Bulk velocity is implemented in a straight forward way by applying an extra boost to the seed photons, i.e., the energy $k$ and angle $\mu$ of an incoming photon in the neutron star frame are transformed to the electron rest frame via [4]

$$
k^{\prime}=(1-\beta \mu) \gamma k \quad \text { and } \quad \mu^{\prime}=\frac{\mu-\beta}{1-\beta \mu}
$$

with $\beta=v$ and $\gamma=1 /\left(1-\beta^{2}\right)$. The energy of the $n$-th resonance $E_{\text {res }}$ in the case of bulk velocity (blue vertical lines in Fig. 1) can be obtained by calculating the energy $k$ in the neutron star frame which if boosted to the electron rest frame matches the resonance energy $E_{\text {res }}^{\prime}$,

$$
E_{\mathrm{res}}^{\prime}=m_{e} \frac{\sqrt{1+2 n B \sin ^{2} \theta}-1}{\sin ^{2} \theta} \quad \text { and } \quad E_{\mathrm{res}}=\frac{E_{\mathrm{res}}^{\prime}}{(1-\beta \mu) \gamma}
$$




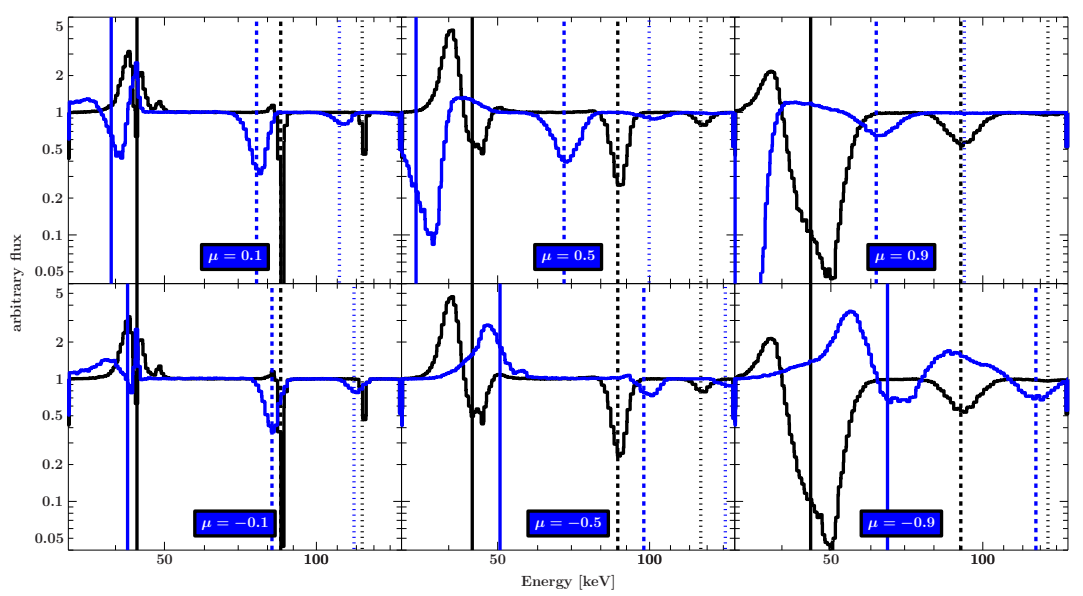

Figure 1. Simulated CRSF spectra for cylinder geometry with a magnetic field of $B=0.09 B_{\text {crit }}$, a temperature $T=3 \mathrm{keV}$, and an optical depth of $\tau=10^{-3}$ for different viewing angles $\mu=\cos \theta$. The black lines show spectra for the case of zero bulk velocity. A bulk velocity of $v=-0.4 c$ was assumed for the blue lines.
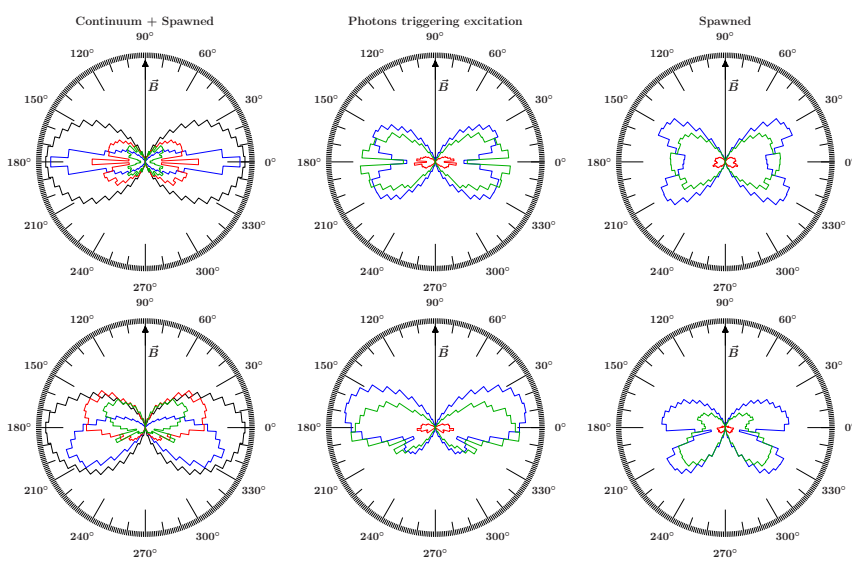

Figure 2. Angular distribution of photons emerging from a static cylinder column (top) and from one with bulk velocity $v_{\text {bulk }}=-0.4 c$ (bottom). From left to right: All escaping photons, photons triggering spawning processes, and escaped spawned photons. The black lines correspond to arbitrary energies while the blue line corresponds to photons with an energy $E^{\prime}$ for which $\left|E^{\prime}-E_{\text {res }, 0}^{\prime}\right|<1 \mathrm{keV}$ showing photons effectively filling up the fundamental cyclotron line. The green and the red lines are the same as the blue line but for the higher resonances $n=1$ (green) and $n=2$ (red). The simulation parameters are the same as in Fig.1.

Fig. 1 shows spectra for different viewing angles to the magnetic field for the symmetric, $v_{\text {bulk }}=0$ (black), case and for the case of bulk motion with velocity $\left|v_{\text {bulk }}\right|=0.4 c$ anti-parallel to the magnetic field (blue). The symmetry between positive and negative $\mu$ for cylinder geometry is broken by the introduction of bulk velocity. For photons escaping the column in the direction of the magnetic field (positive $\mu$ ) the fundamental cyclotron line is much deeper because of the angular redistribution of fundamental line photons towards negative $\mu$ (see Fig. 2). The origin of this change in emissivity is caused by the angular redistribution of scattered photons and by the beaming of spawned photons in the direction of motion. Due to the lack of altered photons parallel to the field the fundamental line can not be filled up resulting in a deep absorption feature in contrast to the emission features observed for slightly negative $\mu$.

\section{Angular redistribution}

The introduction of bulk velocity affects the angular redistribution considerably. Figure 2 shows the final angles of all photons from one simulation run with and without bulk velocity. The additional 
velocity component introduces beaming in two reference frames: First the seed photons injected from the neutron star frame are boosted to the moving plasma's rest frame affecting their energies and angles with respect to the later. Secondary photons spawned by electrons within the moving plasma are boosted into the opposite direction if observed in the neutron star frame. Therefore these spawned photons are beamed downwards in contrast to continuum photons which tend to escape upwards due to the smaller variety of electron momenta in that direction.

\section{Discussion}

Our simulations yield shifted cyclotron resonances depending on the viewing angle and different final angular distributions of the escaping photons for a plasma with a bulk velocity component compared to the static case. The angular redistribution can be understood from analyzing the paths of seed continuum, scattered, and spawned photons independently at different energy bands. Most resonant photons escape perpendicular to the magnetic field effectively filling up the fundamental line in that direction. The behavior of the higher harmonics is different since they allow observations from further within the column due to their smaller cross sections by orders of magnitude compared to the fundamental. The introduction of bulk velocity exposes their difference by the observation of different boosting directions. The photons shown here are all very close to the corresponding resonant condition but in the case of the higher harmonics the photons are continuum photons which managed to escape the column. Since more continuum photons escape in the energy band of Landau level $n=2$, the second harmonic is less deep than the first harmonic as observed in the simulated spectra, see e.g. Fig. 1. Continuum photons escaping upwards encounter a smaller variety of electron momenta and therefore have a better chance of escaping the column. Continuum photons within the fundamental line resonance, on the other hand, have a significantly smaller mean free path. In the regime of optical depth considered here, this smaller path means that they are scattered or absorbed far before the medium's border. The fundamental line photons seen in Fig. 2 show photons scattered of electrons in the ground state and photons spawned by previously excited electrons. The spawning electron's bulk velocity component leads to downward beaming of the spawned photons. This effect is negligible for the higher harmonics since effectively all deexcitations take place to the next lower Landau level producing a fundamental line photon. These beaming patterns have strong impact on the observed pulse profiles. Since the behaviour of the fundamental line and the higher harmonics differ we expect to see similar trends at the corresponding positions in the pulse profiles. The impact of cyclotron angular redistribution on observable X-ray pulse profiles has been investigated recently by [6] including general relativistics and a suitable example case.

\section{References}

[1] P.A. Becker, M.T. Wolff, ApJ654, 435 (2007), arXiv: astro-ph/0609035

[2] H.G. Latal, ApJ309, 372 (1986)

[3] M. Isenberg, D.Q. Lamb, J.C.L. Wang, ApJ505, 688 (1998), arXiv : astro-ph/9703075

[4] R.A. Araya, A.K. Harding, ApJ517, 334 (1999)

[5] G. Schönherr, J. Wilms, P. Kretschmar, I. Kreykenbohm, A. Santangelo, R.E. Rothschild, W. Coburn, R. Staubert, A\&A472, 353 (2007), 0707.2105

[6] G. Schönherr, F.W. Schwarm, S. Falkner, T. Dauser, C. Ferrigno, M. Kühnel, D. Klochkov, P. Kretschmar, P.A. Becker, M.T. Wolff et al., A\&A(2013), submitted 